\title{
Nivel de preocupación o ansiedad sobre procedimientos dentales en una sala de espera dental
}

RICARDO MUZA ${ }^{(1)}$ Y PAULA MUZA(2)

\section{RESUMEN}

Su objetivo es conocer el nivel de preocupación o ansiedad sobre procedimientos dentales en una sala de espera dental. Para ello, se aplicó la encuesta Dental Concerns Assessment (DCA), traducida, en la sala de espera del Servicio Dental del Hospital Regional Rancagua a 233 pacientes mujeres, acerca de 26 procedimientos dentales. La escala de medición de la ansiedad tiene 4 niveles (Bajo, Moderado, Alto, No sabe) La edad fue clasificada en 4 grupos etarios por los que se compararon las respuestas.

Resultados: Los mayores niveles de preocupación estuvieron causados por el costo de los tratamientos (156 pacientes), la necesidad de varios procedimientos (131), el número de citaciones y el tiempo asociado (118), el dolor (117), temor a la inyección (111), tratamiento de endodoncia (104) y por el sonido del aparato empleado en la limpieza dental (96). El procedimiento que causó menos ansiedad es la radiografía (26).

El uso de cuestionarios de evaluación de la ansiedad pre-tratamiento, puede ayudar a los dentistas a disminuir la ansiedad de sus pacientes. Es necesario tomar medidas concretas para manejar la ansiedad, tales como, fármacos, técnicas de relajación, respiración abdominal, meditación, música.

Palabras claves: Ansiedad, preocupación, procedimientos dentales.

\section{ABSTRACT}

DEGREE OF CONCERN OR ANXIETY ABOUT DENTAL PROCEDURES AT A DENTAL WAITING ROOM

The purpose of the present work is to assess the level of concern or anxiety about dental procedures at a dental waiting room. To achieve such purpose, the translated Dental Concerns Assessment (DCA) survey was administered to 233 patients at the waiting room of the Dental Service of the Hospital Regional Rancagua. The survey assessed 26 dental procedures. The anxiety scale had 4 levels (low, moderate, high, does not know). The age of the patients was stratified into 4 age groups, and the answers were compared between age groups.

Results: the highest levels of concern were caused by the cost of therapies (156 patients), the need of more than one procedure (131), the number of visits and time related to these (118), pain (117), fear of the shot (111), endodontic therapy (104) and by the sounds of the device used in dental cleaning (96). The procedure causing the less anxiety was $x$-rays (26).

\footnotetext{
(1) Servicio dental, Hospital Regional Rancagua.rmuzac@yahoo.com

(2) Psicóloga Clínica.
} 
The use of questionnaires to assess pretreatment anxiety may help dentists to decrease their patients' anxiety. Consistent measures are needed to manage anxiety, such as drugs, relaxation techniques, abdominal breathing, meditation and music.

Keywords: Anxiety, concern, dental procedures.

\section{INTRODUCCION}

La mayor parte de la población en Chile tiene una salud bucal deteriorada que necesita ser atendida. Esta realidad se ve empeorada por la ansiedad o temor que sienten un porcentaje de los pacientes previo a la atención; debido a ello muchos evitan o postergan su atención dental, con consecuencias peores. Por ello, es necesario romper el círculo que produce el temor o ansiedad, que lleva a evitar los cuidados dentales y el deterioro consiguiente de la salud bucal que además produce otros efectos indeseables como sentimiento de culpa, vergüenza y sentimiento de inferioridad. En este estudio hablaremos de temor y ansiedad dental, como sinónimos.

La ansiedad dental es muy importante, es el principal componente del sufrimiento/angustia en los pacientes de operatoria dental ${ }^{1}$. A pesar del avance tecnológico en equipamientos dentales, la ansiedad y el temor al dolor están presentes frente a un tratamiento dental. Estudios epidemiológicos sugieren que entre el $3 \%$ y $20 \%$ de la población tienen niveles de temor y ansiedad acerca del tratamiento dental, lo que es problemático ${ }^{2}$. Al buscar atención dental, la ansiedad está presente, y a veces llega a ser difícil de manejar al sentarse en el sillón dental. Evitar el tratamiento dental debido a la ansiedad es muy común y puede estar fuertemente asociado con el deterioro de la salud oral y dental ${ }^{3}$. En una encuesta en la población de Estados Unidos se encontró que aproximadamente el $15.5 \%$ de quienes la respondieron, tenían grados de temor dental y evitaban la atención ${ }^{4}$. También uno de cada 8 adultos británicos tenía ansiedad dental asociada con su uso de los servicios y su estado de salud oral $^{4}$. La ansiedad dental varía de un paciente a otro. Está ampliamente difundido que decrece con la edad; las razones sugeridas incluyen diferencias de cultura y experiencias en los grupos etarios; lo cual puede ser debido a que la ansiedad, en general, disminuye con la edad ${ }^{5}$.

No todos los procedimientos o etapas de un tratamiento dental producen el mismo nivel de ansiedad. Aquellos que generan altos niveles de ansiedad son: extracciones, preparación de cavidades, o mirar la jeringa y la aguja sostenida por el dentista. Wong y Lytle encuentran que la extracción dental y los tratamientos de endodoncia son los procedimientos que generan mayor ansiedad dental ${ }^{6}$.

El dentista debe estar conciente que los tratamientos dentales provocan cierto grado de ansiedad, y debe estar preparado para enfrentarlos, tomando medidas concretas para evitar o disminuir la ansiedad.

En el Servicio Dental del Hospital Rancagua se entrega atención a los pacientes en diferentes especialidades odontológicas, con distintos tipos de procedimientos dentales. Por ello es importante conocer su nivel de ansiedad o preocupación antes de ingresar a la clínica dental. Ello nos motivó a realizar el presente estudio. Se hace necesario aplicar todos los instrumentos que permitan disminuir la tensión, tales como, técnicas de relajación, respiración abdominal profunda y lenta, apoyo con música suave, y si fuese necesario, apoyo medicamentoso farmacológico.

Los tratamientos dentales pueden ser una experiencia estresante para muchos pacientes como también para los dentistas. Los síntomas más comunes de los pacientes ansiosos son la respiración pesada, agitada, transpiración, movimientos de manos, sudoración. La mayoría de los dentistas no aplican herramientas para medir la ansiedad de los pacientes, previo a ser 
sometidos a tratamientos, y en consecuencia no aplican medidas para manejarla adecuadamente.

El tiempo prolongado previo a la atención dental, el trato inadecuado del dentista por mal carácter o brusquedad, son factores que suscitan temor en los pacientes. Se ha comprobado que la angustia que despierta el tratamiento odontológico es de tipo emocional, pues es previo a recibir la atención correspondiente. A veces pueden aparecer en pacientes que no han experimentado dolor ni situaciones desagradables o traumáticas anteriores; no obstante, la han aprendido por asociación de terceros.

\section{OBJETIVO}

Conocer el nivel de ansiedad o preocupación de pacientes mujeres en una sala de espera dental, con relación a procedimientos dentales.

\section{MATERIAL Y MÉTODO}

Es un estudio descriptivo, realizado durante los meses de enero a enero a marzo de 2006, a 233 pacientes mujeres mayores de 16 años, que esperaban atención dental en la sala de espera del Servicio Dental del Hospital Rancagua y que aceptaron voluntariamente responder el cuestionario, "Dental Concerns Assessment" (DCA), que mide la ansiedad frente a 26 procedimientos o acciones dentales. La muestra tiene el sesgo de haber sido aplicada solamente a mujeres, y no de forma aleatoria; sino que se aplicó a las pacientes que aceptaron contestarla, que fue aproximadamente el $92 \%$ de quienes fueron requeridas. El cuestionario fue aplicado por un alumno de $\mathrm{V}$ año de Odontología que realizaba su práctica hospitalaria y que fue capacitado para la aplicación de la encuesta, validada previamente en 30 pacientes mujeres en las mismas condiciones. No existen antecedentes bibliográficos que este cuestionario haya sido aplicado anteriormente en Chile. Los pacientes tenían que responder si su nivel de ansiedad o preocupación, frente a las 26 preguntas, era: bajo, medio, alto, no sabe. Los datos recogidos fueron analizados estadísticamente mediante el programa SPSS.

\section{RESULTADOS}

Total de pacientes encuestados $233 ; 22$ pacientes $(9.4 \%)$ son de 16 a 20 años; 136 (58.4\%) de 21 a 40 años; 66 (28.3\%) de 41 a 60 años; y 9 (3.9\%) son de 61 y más años.

De las 233 pacientes que respondieron, se encontró que los mayores niveles de ansiedad o preocupación (catalogado como alto) fueron: Estoy preocupada por el costo del tratamiento dental que puedo necesitar (156); Estoy preocupada porque pueda necesitar varios tratamientos dentales (131); Estoy preocupada por el número de citaciones y el tiempo requerido para ello (118); Miedo por el dolor (117); Inyección (111); Tratamiento de endodoncia (104); Cansancio mandibular, por tener la boca abierta(100); sonido de aparato de limpieza dental (96). La menos mencionada como de alta preocupación o ansiedad fue: toma de radiografía (26).

\section{DISCUSION}

Con relación a los grupos etarios de la muestra en estudio, el $86.7 \%$ tenían de 21 a 60 años de edad. Figura 1

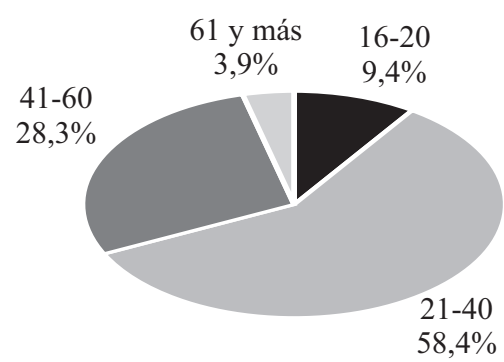

Figura 1. Distribución por edad

Este tema es poco estudiado en Chile, lo que representa una limitación de este estudio; pero a la vez es un aliciente para desarrollar estudios de este tipo a nivel nacional con 
muestras que incluyan mayor cantidad de pacientes, y ojalá de ambos sexos. El departamento odontológico, en la página web del Ministerio de Salud, está conciente de la importancia de la ansiedad que provoca la atención dental. Varias investigaciones han demostrado que las mujeres tienen mayores niveles de ansiedad frente a los diferentes tratamientos o procedimientos dentales. Las mujeres reportan mayores niveles de ansiedad frente a estímulos específicos (inyecciones, agujas) de tratamientos dentales ${ }^{7}$. Estos resultados son consistentes con la literatura de psicología, la cual muestra que las mujeres expresan mayores niveles de temor que los hombres $^{8}$. Este estudio tiene la limitación que fue realizado sólo en mujeres, por lo tanto no se puede hacer una comparación con hombres.

El dentista debe estar conciente de la ansiedad del paciente, para poder manejarla. La interrelación dentista-paciente, es importante para reducir la ansiedad del paciente y para mejora la satisfacción del paciente con relación al tratamiento recibido9. El trato amable, acogedor, y calmo ayuda a disminuir los temores de los pacientes. Dentistas en asociación con psicólogos han estado buscando métodos para detectar y reducir la ansiedad de los pacientes dentales, tales como, la relajación y reestructuración cognitiva. ${ }^{10}$. Sin embargo los dentistas, a menudo, no tienen tiempo o no están preparados para usar métodos de intervención complejos en esta área ${ }^{11}$.

Los resultados de este estudio, realizado con pacientes del sistema público, muestran que las pacientes manifiestan el mayor nivel de ansiedad o preocupación, por los costos de los tratamientos dentales; en un $67.0 \%$ de las pacientes, siendo menor la preocupación (31.8\%) en las pacientes de 16 a 20 años. La preocupación por la necesidad de varios tratamientos dentales es alto, en un $56,2 \%$ de las pacientes, siendo menor la preocupación en las pacientes mayores de 61 años $(33.3 \%)$. La preocupación por el número de citaciones y el tiempo necesario para el tratamiento, por su trabajo o por los niños, es alto en un $50.2 \%$ de las pacientes, siendo similar en todos los grupos etarios. Llama la atención que la preocupación manifestada por la inyección, se presenta sólo en el $47.6 \%$ de las pacientes, siendo menor en el grupo de mayores de 61 años $(33.3 \%)$. La preocupación por tratamiento de endodoncia (tratamiento de conducto) es alto, $44.6 \%$ de las pacientes de la muestra, siendo similar en todos los grupos etarios. Una explicación posible a estos resultados encontrados podría ser, que los ingresos económicos de las pacientes que acuden al sistema público son tan bajos, que les preocupan más los costos y el tiempo involucrado en la atención dental, que una inyección o un tratamiento de endodoncia. En cambio, en países con ingresos más altos, las preocupaciones mayores no pasan por los costos involucrados sino por la acción misma de la atención dental, por ejemplo inyección o tratamiento de endodoncia.

Era esperable que el procedimiento dental que causara menor nivel de ansiedad o preocupación sea la radiografía (11.2\%), siendo menor $(0 \%)$ en el grupo de mayores de 61 años, ya que es un procedimiento indoloro, con aparato externo, y conocido.

El uso de cuestionarios de evaluación de la ansiedad pre-tratamiento, como el "Dental Concerns Assessment" puede ayudar a los dentistas a manejar la ansiedad de sus pacientes. Es necesario tomar medidas concretas para disminuir la tensión, tales como, fármacos, relajación, respiración abdominal, meditación, música.

Las pacientes tienen niveles de preocupación más alto, por situaciones que rodean a la atención misma, como son los costos y el tiempo involucrado, que por los procedimientos terapéuticos dentales propiamente tales. Los dentistas deben tomar conciencias de estas situaciones, y mejorar sus habilidades en el manejo de la entrega de la atención.

\section{REFERENCIAS}

1. CORAH NL, GALE EN, ILLIG SJ. Assessment of a dental anxiety scale. J Am Dent Assoc 1978; 97 : 816-819.

2. MILGROM P, FISET L, MELNICK S, ET AL. The 
prevalence and practice Consequences of dental fear in a major US city. J Am Dent Assoc 1983; 116: 641647.

3. GATCHEL RJ, INGERSOLL BD, BOWMAN L, ET Al. The prevalence of dental fear and Avoidance: a recent survey study. J Am Dent Assoc 1983; 107: 609-610.

4. BEDI R, MCGRATH C. Factors associated with dental anxiety among older people in Britain. Gerodontol 200; 17: 97-103

5. LOCKER D, LIDDELL AM. Correlates of dental anxiety among older adults. J Dent Res 1991; 70 : 198-203

6. WONG M, LYTLE WR. A comparison of anxiety levels associated with root canal therapy and oral surgery treatment. J Endod 1991; 17: 461-465

7. HOLTZMAN J, BERG R, MANN J, BERKEY D. The relationship of age and gender to fear and anxiety response to dental care. Spec Care Dentist 1997; 17:
82-87

8. BRINK-MUINEN A, BENSING J, KERSSENS J. Gender and Communication Style in General Practice: Differences Between Women's Health Care and Regular Health Care. Med Care 1998; 45: 520-524

9. CORAH NL. Dental anxiety. Assessment, reduction and increasing patient satisfaction. PMID: 3053270 (PubMed-indexed for MEDLINE)

10. GETCHEL RJ (1980). Effectiveness of two procedures for reducing dental fear: groupadministered desensitization and group education and discussion. The Journal of the American Dental Association, 101, 634-637

11. MILGROM, P., WEISTEIN, P., KLEINKNETCH, R., \&GETZ, T. (1985). Treating fearful dental patients. Virginia: Prentice Hall, Inc.

Recepción:18 dic. 2006 Aprobación:5 abr. 2007

Usted puede comentar éste y otros artículos publicados en la Revista Chilena de Salud Pública, enviando un correo electrónico a revistasp@med.uchile.cl 